\title{
Assessing the Impact of Unbalance in Data on Predicting Breast Cancer Occurrence Using Machine Learning Models
}

\author{
Shuning Yin, Raji Sundararajan* and Gaurav Nanda \\ School of Engineering Technology, Purdue University, West Lafayette, USA \\ *Corresponding Author: Raji Sundararajan, School of Engineering Technology, \\ Purdue University, West Lafayette, USA.
}

Received: December 03, 2021

Published: January 25, 2022

(C) All rights are reserved by Raji

Sundararajan., et al.

\begin{abstract}
With over 2 million new cases and over 600,000 deaths each year, breast cancer is the most common cancer among women all over the world. Early detection and treatment can save 1000s of lives. To achieve this goal, we used the machine learning library, WEKA and the Breast Cancer Surveillance Consortium (BCSC) dataset with 154,899 screening records. This dataset has twelve variables, and we considered the variable "breast_cancer_history", as the main variable to be predicted. The various machine learning (ML) classifiers examined include Naïve Bayes, Logistic Regression, Multilayer Perceptron, and Support Vector Machine with five different test conditions. Since the dataset had less cancer cases ("Class 1"), compared to non-cancer (heavily unbalanced with very high percentage of "Class 0 ") cases, we examined the prediction performance of these algorithms on "balanced" and "unbalanced" datasets. Stratified sampling method was used to create unbalanced and balanced datasets. The unbalanced dataset included all the original screening data (154,899 cases) and the balanced dataset included the same number of cases for both class 0 and class 1 $(13,279$ each). Of the four ML classifiers, Multilayer Perceptron had the best predicting performance for both unbalanced and balanced dataset. Overall, the balanced dataset had better prediction results for all four classifiers than the unbalanced dataset. For medical decision support purposes, we can use the prediction outputs from the ML models, trained on both balanced and unbalanced training data.
\end{abstract}

Keywords: Breast Cancer; Machine Learning; Data Analysis; WEKA; Prediction

\section{Introduction}

Breast cancer is the most common cancer among women worldwide and also one of the leading causes of cancer death in women [1]. In the US, in 2014, about 237,000 women were diagnosed with breast cancer and 41,211 women died of it [2,3]. Each year, the American Cancer Society estimates the numbers of new cancer cases and deaths that will occur in the United States in the current year and compiles the most recent data on cancer incidence, mortality, and survival. Incidence data were collected by the National Cancer Institute (Surveillance, Epidemiology, and End Results [SEER] Program. In 2021, it is estimated that there will be 281,550 new cases and 43,600 deaths [4]. About 1 in 8 U.S. wom- en have a risk of developing breast cancer during her lifetime [5]. Studies have shown that old age is a risk factor for breast cancer, after developing an age-period-cohort analysis of the 1999-2014 breast cancer data $[5,6]$.

Currently, a number of data sets are collected for cancer diagnosis and detection and the utilization of machine learning in cancer research may be considered as an effective approach [7-11]. This is because, machine learning, a subfield of artificial intelligence (AI) employs many algorithms that allow computers to learn from training data and develop a fitted pattern for prediction is more commonly used to predict the breast cancer patterns [12-14], biology, finance, and marketing. The challenge of understanding 
these data has led to the development of new tools in the field of statistics, and spawned new areas, such as data mining, machine learning, and bioinformatics. Pironet., et al. used machine learningbased extraction of breast cancer receptor status from bilingual free-text pathology reports. This involved reports from 82 labs in two national languages (Dutch and French) to study the molecular features of breast cancer [15]. Ming., et al. used machine learning techniques for personalized breast cancer risk prediction. They compared these models with the Breast Cancer Risk Assessment Tool (BCRAT) and Breast and Ovarian Analysis of Disease Incidence and Carrier Estimation Algorithm (BOADICEA) models and found significant improvement in the accuracy of classification of women with and without breast cancer using ML models [16].

Asri., et al. implemented four types of machine learning methods, including SVM, Decision Tree, Naive Bayes and k Nearest Neighbors (k-NN) on the original Wisconsin Breast Cancer datasets using the software WEKA to find the most efficient method for breast cancer prediction and diagnosis. They compared the effectiveness and efficiency of the four algorithms based on accuracy, precision, sensitivity and specificity and reported that SVM outperformed with the best classification accuracy of $97.13 \%$ and lowest error rate among all the methods [17]. Li., et al. also utilized the BCSC in their machine learning-based decision support system study for breast cancer early detection. Five machine learning algorithms, including Logistic Regressions, Naïve Bayes, Decision Trees, k-NN as well as SVM were used. They trained the methods with two conditions: ten-fold cross-validation approach and 80\% split and evaluated the performance based on the measurements of sensitivity and specificity. Their study found out that SVM performed the best while Logistic Regressions, Naïve Bayes and Decision Trees performed quite close [18]. Ahmad., et al. developed models for predicting breast cancer recurrence. They used the medical data from the Iranian Center for Breast Cancer (ICBC) and applied three machine learning algorithms, Decision Tree, SVM, and Artificial Neural Network (ANN), with 10-fold cross-validation test condition. Their study concluded that SVM classification model performed the best on breast cancer recurrence prediction with the highest accuracy (95.7\%) and least error rate among all algorithms
[19]. This research presented a comparison among three machine learning algorithms applied in the breast cancer dataset: support vector machine (SVM), naive bayes classifier, and logistic regression by measuring their accuracy, sensitivity, and specificity values.

In our study, we used supervised learning models for assessing the impact of unbalance in the data on predicting breast cancer occurrence using four ML models.

\section{Methods}

\section{Dataset}

The study used the BCSC dataset [20], with records observed from 2000 to 2009 calendar year. The dataset contained various biographic and physiological information about the individuals in separate fields, as listed in table 1 [20], with possible values for each field.

As shown in Table 1, this dataset has thirteen fields. We chose the field, "breast_cancer_history" (prior breast cancer diagnosis) as the dependent variable to be predicted based on the values of other fields, which were considered as independent variables. Since the variable "year" only indicated the year of record creation and did not provide any meaning or information about the patient's health or background, it was disregarded from further analysis. The eleven fields considered include "age_group_5_year" (age in 5 year groups), "race_eth" (race/ethnicity), "first_degree_hx" (history of breast cancer in a first degree relative), "age_menarche" (age at menarche), "age_first_birth" (age at first birth), "BIRADS_breast_ density" (BI-RADS breast density), "current_hrt" (use of hormone replacement therapy), "mebnopaus" (menopausal status), "bmi_ group" (body mass index), "biophx" (previous breast biopsy or aspiration), and "count" (frequency count of this combination of covariates). It is to be noted from table 1 that only the field "count" was a numerical variable, and all the other fields/variables were nominal in nature.

For the field, "breast_cancer_history", the value 0 meant negative breast cancer diagnosis, value 1 meant positive breast cancer diagnosis, and value 9 meant status unknown. The original BCSC 


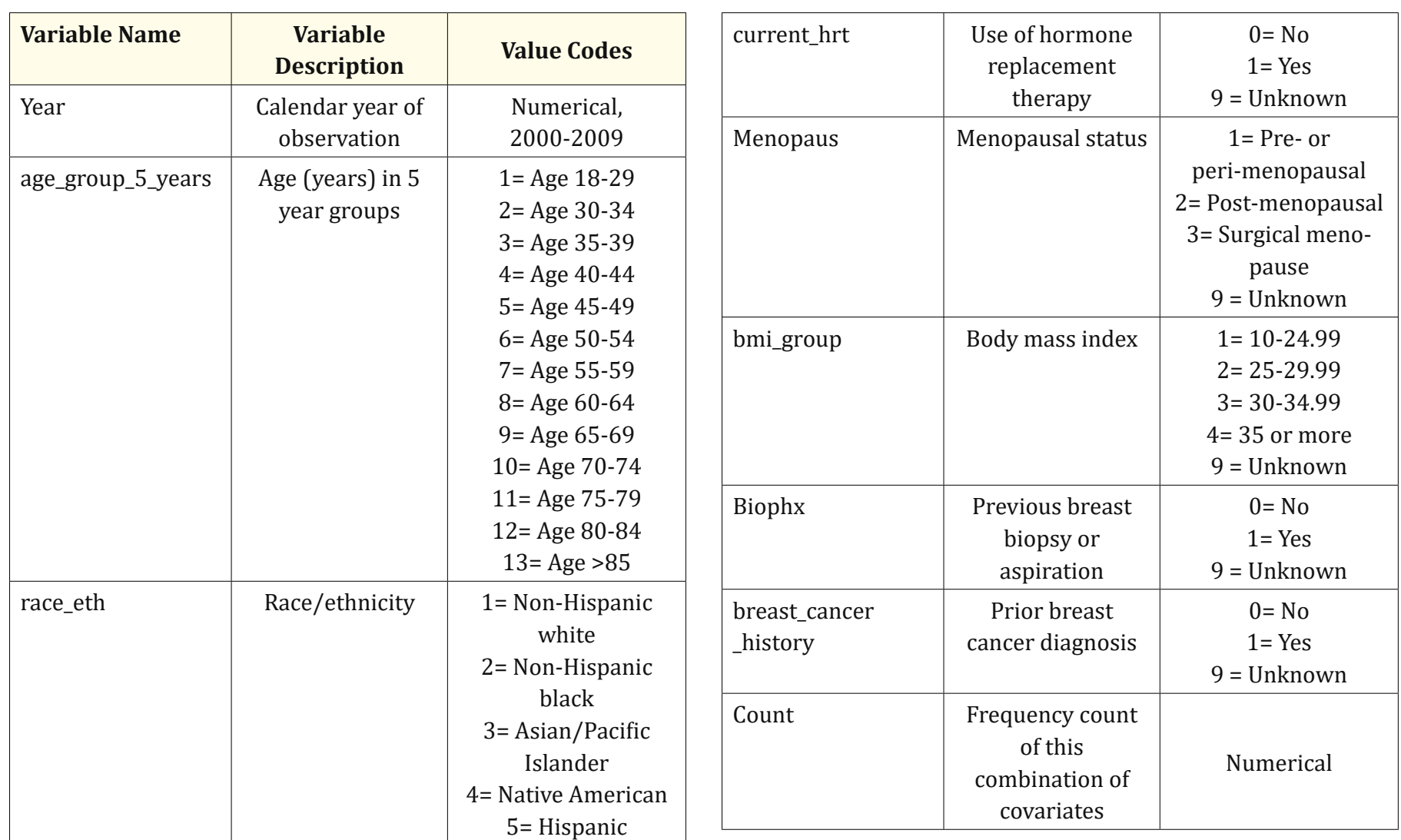

Table 1: Variables of breast cancer from BCSC. $6=$ Other $/$ mixed $9=$ Unknown

\begin{tabular}{|c|c|c|}
\hline first_degree_hx & $\begin{array}{c}\text { History of breast } \\
\text { cancer in a first } \\
\text { degree relative }\end{array}$ & $\begin{aligned} & 0=\text { No } \\
& 1=\text { Yes } \\
& 9= \text { Unknown }\end{aligned}$ \\
\hline age_menarche & $\begin{array}{l}\text { Age (years) at } \\
\text { menarche }\end{array}$ & $\begin{array}{c}0=\text { Age }>14 \\
1=\text { Age } 12-13 \\
2=\text { Age }<12 \\
9=\text { Unknown }\end{array}$ \\
\hline age_first_birth & $\begin{array}{c}\text { Age (years) at first } \\
\text { birth }\end{array}$ & $\begin{array}{c}0=\text { Age }<20 \\
1=\text { Age } 20-24 \\
2=\text { Age } 25-29 \\
3=\text { Age }>30 \\
4=\text { Nulliparous } \\
9=\text { Unknown }\end{array}$ \\
\hline $\begin{array}{l}\text { BIRADS_breast__ } \\
\text { density }\end{array}$ & $\begin{array}{c}\text { BI-RADS breast } \\
\text { density }\end{array}$ & $\begin{array}{c}\text { 1= Almost entirely } \\
\text { fat } \\
2=\text { Scattered fibro- } \\
\text { glandular densities } \\
3=\text { Heterogeneously } \\
\text { dense } \\
\text { 4= Extremely dense } \\
9=\text { Unknown or } \\
\text { different measure- } \\
\text { ment system }\end{array}$ \\
\hline
\end{tabular}

dataset contained 1,144,565 records. From the original dataset, we removed the records where the following condition were TRUE:

\{breast_cancer_history $=9\}$ OR $\{$ count $<5\}$

This was done in order to prevent the machine learning model to learn from cases, where the predicted variable breast_cancer_ history was unknown or the count of the combination of values was very uncommon $(<5)$. After removing these cases, our final dataset contained 154,898 cases, with 141, 619 cases with breast_ cancer_history $=0$ and 13,279 cases with breast_cancer_history = 1. Reflective of the real-world, the BCSC dataset had a very small percentage of positive cancer cases (9\%) compared to non-cancer cases $(91 \%)$. Such a heavily skewed distribution of categories is very challenging for machine learning models and often leads to poor prediction of the smaller category [21]. In this case, therefore, it was expected that it would be extremely challenging to obtain a good prediction performance for the positive breast cancer cases using machine learning models. To tackle this, we explored the four ML models. 
Machine learning models

Based on the data types used to train in the experiments, there are three main categories of machine learning: supervised learning, unsupervised learning, and reinforcement learning [12,22]. The machine learning algorithms used in this study were all supervised learning methods, trained by labeled data [12,22,23]. Supervised machine learning models can be trained on hand-labeled historical data to predict outcomes or classify information for new data and can thus help humans in decision making [22]. In this work, the following four supervised ML models were used: Naïve Bayes, Multilayer Perceptron (MLP), Logistic Regression, and Support Vector Machine (SVM).

\section{Naïve bayes}

The Naïve Bayes method is a classification method, based on the Bayes theorem and feature condition independent hypothesis. For a given training dataset, the joint probability distribution of the input/output is first learned, based on independent assumptions of feature conditions; then, it uses Bayes theorem to find the output with the largest posterior probability for a given input. Based on this assumption, the Bayesian formula can be rewritten as . Here, $d$ is the number of features, is the value of feature [8,12-13].

\section{Multilayer perceptron}

The Multilayer Perceptron (MLP) classifier is a type of feedforward artificial neural networks (ANN), which can generate a group of output variables, based on a group of input variables. As the name "multilayer" implies, a MLP consists of three types of layers: input layers, output layers and hidden layers. Input layers receive and process the input data; output layers perform classification and prediction; and hidden layers are connected in between the input and output layers to do the computations. MLPs train the data using back propagation algorithm and are designed to approximate continuous functions and solve non-linear problems [23,24]. In this study, the hidden layers of the MLP algorithm identified each independent variable alone; and later jointly functioned within the neural networks to predict the dependent variable "breast_cancer_ history" and make decisions.

\section{Logistic regression}

Logistic Regression is a classical statistic model to make predictive regression analysis when the dependent variable is in binary. This method is mainly utilized to describe and explain the relationship between one dependent binary variable and one or more other different types of independent variables. Basically, the algorithm works to predict the output variable by using coefficients and weights to linearly combine input variables [12,22]. Logistic regression uses similar equation like linear regression with the key difference which the output value of logistic regression being modeled is binary not numeric. The fitted logistic regression model is represented by the conditional probability distribution with the logistic function:

$$
P(Y=1 \mid x)=\frac{\exp (w x)}{1+\exp (w x)} \text { and } P(Y=0 \mid x)=\frac{1}{1+\exp (w x)}
$$

With $\log \frac{P(Y=1 \mid x)}{1-P(Y=1 \mid x)}=w x$, the log odds of can be represented by the linear function of input $x[12,26]$. In this study, logistic regression algorithm was used to find the relationship between the dependent variable "breast_cancer_history" and other eleven nominal independent variables to make predictions.

\section{Support vector machine}

Support Vector Machine (SVM), also known as support vector network (SVN), is also a binary classifier of machine learning algorithm to make classification and regression analysis. This classifier plots all the data (each data as a point) in a n-dimensional space and sorts the data into two categories by finding the hyper-plane in the plot which can well separate the data to two classes; and builds the model by training the classified data. The SVM algorithm outputs the sorted training data with the largest possible margin between the two classes of it. This method can solve both linear and non-linear problems $[12,25]$.

\section{Performance evaluation}

We utilized the machine learning library, WEKA to apply the four ML models and analyze the prediction performance. To check the performance of the models, the four classifiers were run and modeled in five different training and testing conditions: Crossvalidation Folds 10, Percentage split 60\%, Percentage split 70\%, Percentage split $80 \%$, and Percentage split 90\%.

We used Sensitivity (also known as Recall) and Positive Predictive Value (PPV, also known as Precision), defined below, as the primary performance measures for evaluating prediction performance of individual categories class 0 and class 1 [22].

$$
\begin{aligned}
& \text { Sensivity }=\frac{\text { True Positives }}{\text { True Positives + False Negatives }} \\
& \text { PPV }=\frac{\text { True Positives }}{\text { True Positives + False Positives }}
\end{aligned}
$$




\section{Tackling bias in data}

As indicated earlier and shown in figure 1, the BCSC dataset has a heavily skewed distribution of categories with $91 \%$ cases belonging to Class 0 (negative cancer diagnosis) and $9 \%$ cases belonging to Class 1 (positive cancer diagnosis), just like in the real world.

\section{Data Distribution of Categories}

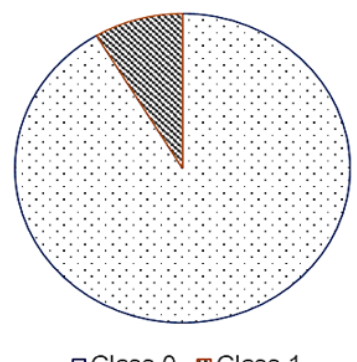

Figure 1: Unbalanced Dataset (Original Dataset).

This situation is also referred as the class unbalance problem in machine learning, where, due to the bias in data, the prediction accuracy for smaller category usually suffers both in terms of recall and precision. In practical terms, it would mean in our case that the machine learning models would not be able to identify potential cases of positive cancer accurately.

To study the impact of class unbalance, we examined the prediction performance of the above four algorithm models, based on "balanced" and "unbalanced" datasets. Stratified sampling method was used to create unbalanced and balanced datasets. The unbalanced dataset was the original dataset, which has all the screening data (154,899 cases). The balanced dataset (Figure 2), included the same number of cases for both class 0 and class $1(13,279$ each, 26,558 cases total).

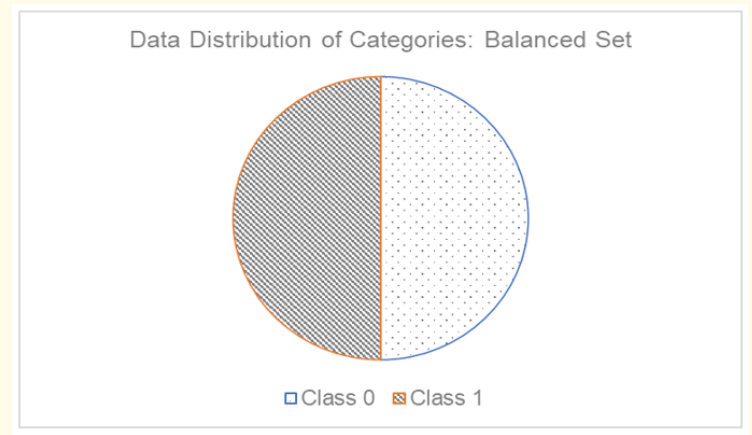

Figure 2: Balanced Dataset.
Figure 3 shows the workflow and the several analyses performed in this study for further and deeper analysis of the bias issue in the data. As shown, we examined the prediction performance of four well-established machine learning models under different testing conditions for balanced and unbalanced datasets to understand how the bias in BCSC data can affect the prediction performance of various machine learning algorithms. The smaller category among the two - Class 1 had a very small percentage of cases in the unbalanced dataset but had a sizeable percentage of cases in the balanced dataset. Our hypothesis was that the prediction performance of Class 1 would be considerably better in the case of balanced dataset as compared to the unbalanced dataset for all machine learning models.

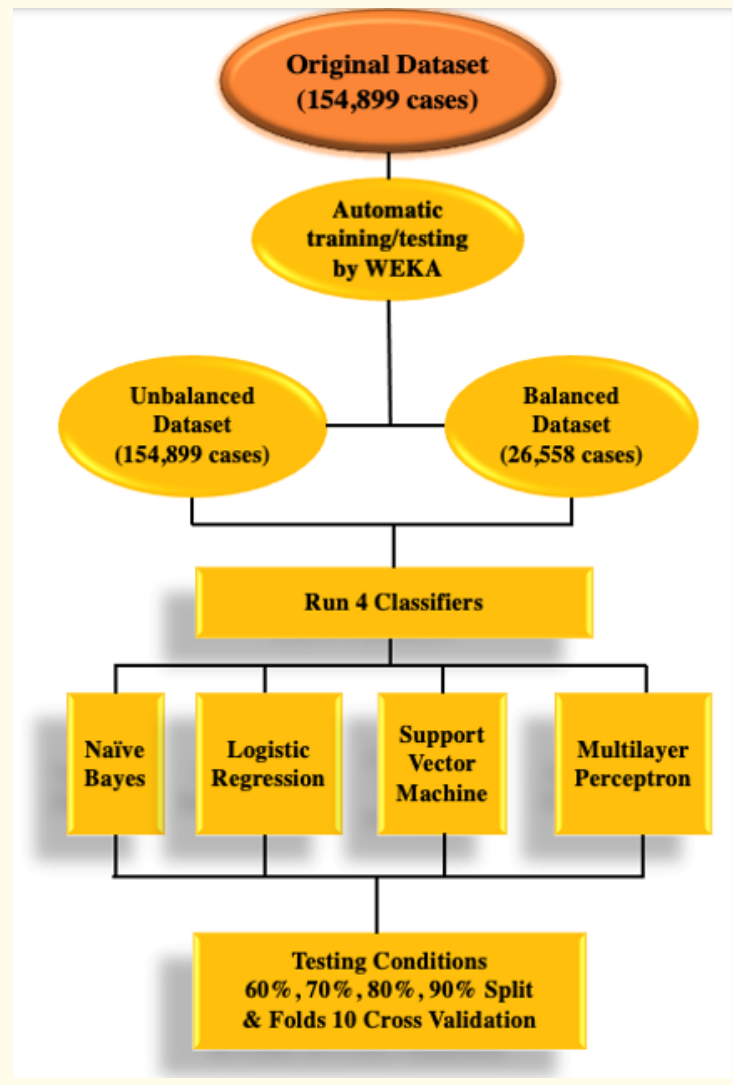

Figure 3: Flowchart of the various experiments performed in this study.

\section{Results and Discussion}

Figures 4 and 5 show the correlation heatmap of the variables of balanced and unbalanced datasets respectively, highlighting how bias impacts the correlation between different variables. Here, 
the level of correlation is different between the same variables in balanced and unbalanced datasets. For example, the correlation value between breast_cancer_history and age_group is higher for balanced dataset as compared to unbalanced dataset. This indicates that the predictive machine learning models developed for balanced and unbalanced datasets will be different.

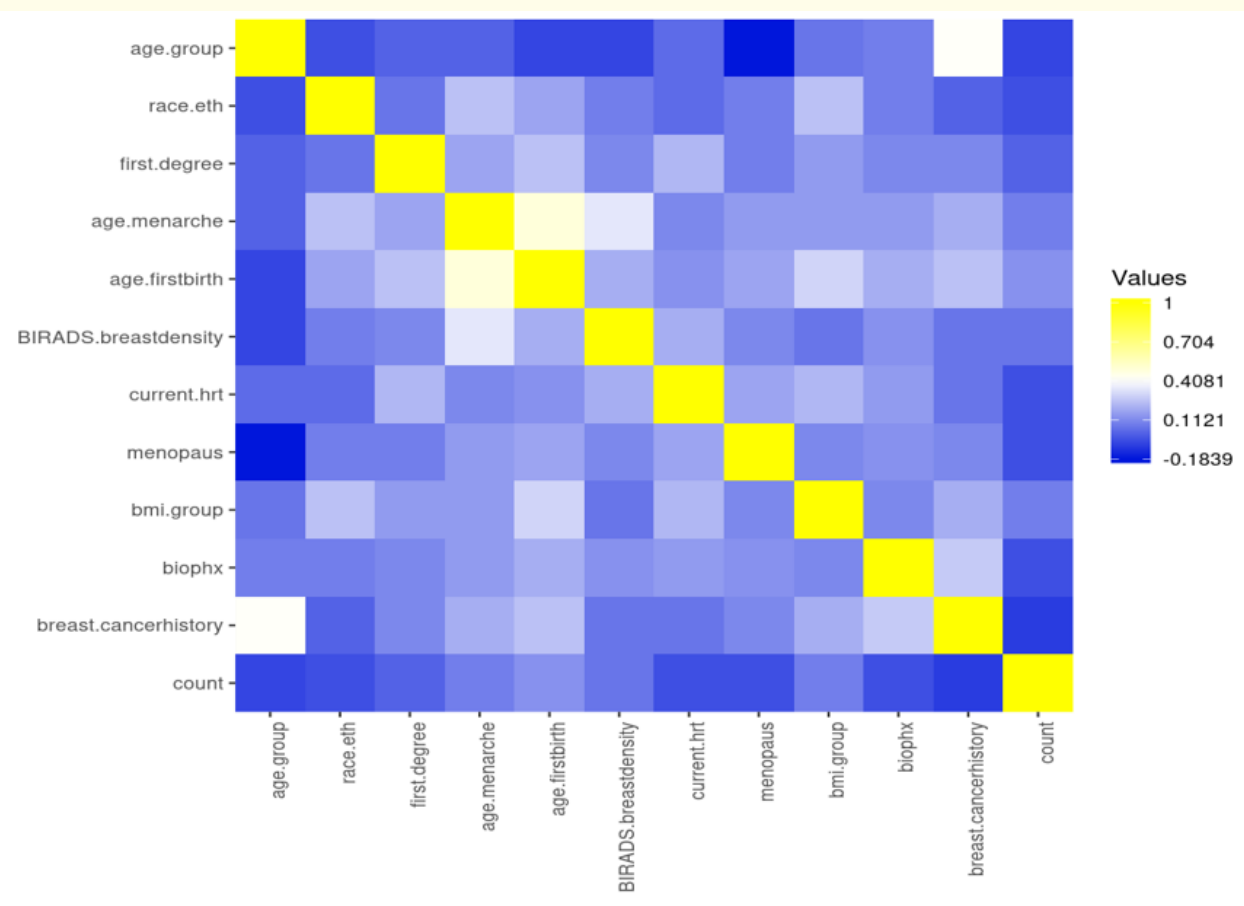

Figure 4: Correlation heatmap of different variables for balanced dataset

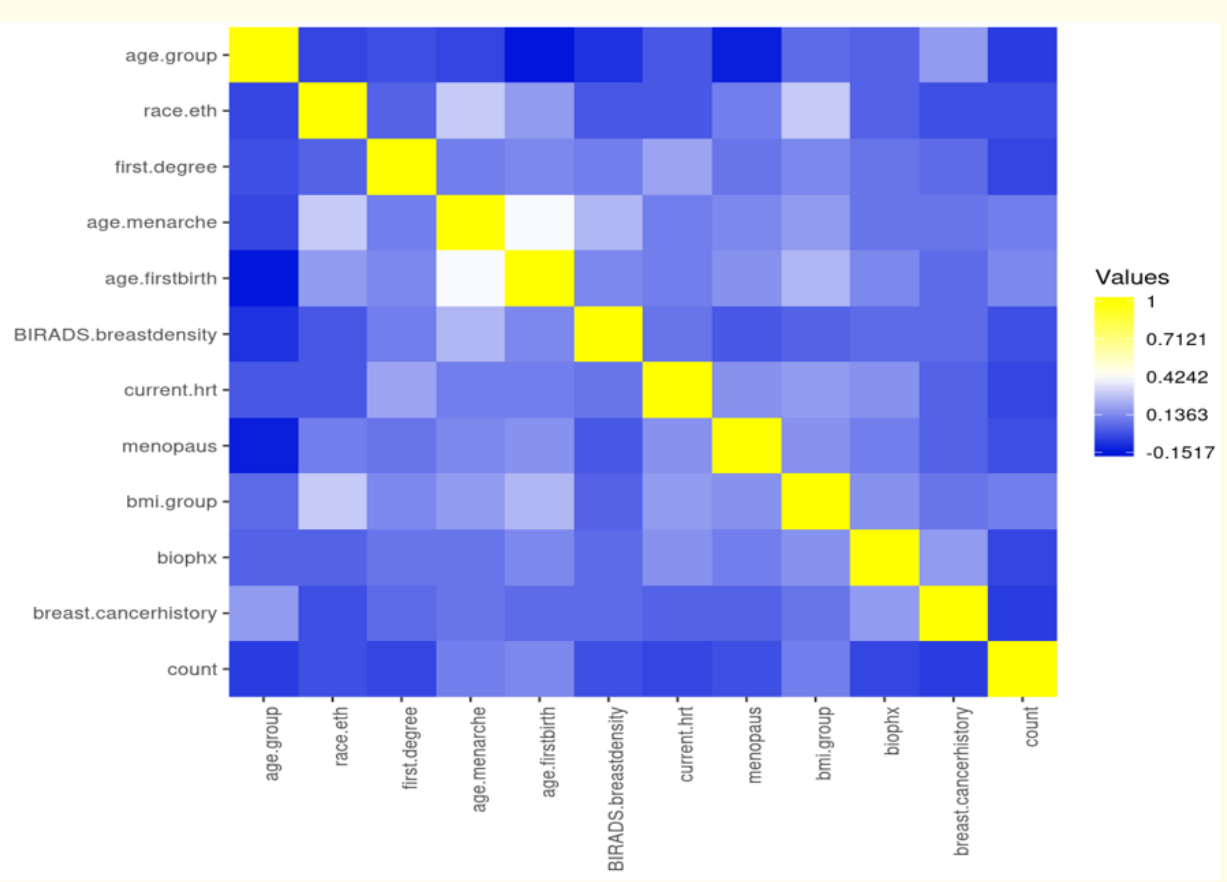

Figure 5: Correlation heatmap of different variables for unbalanced dataset. 
Naïve bayes

The performance measures, Sensitivity and Specificity were recorded from the WEKA result output to evaluate the prediction performance of each model under different testing conditions. A representative screenshot of WEKA result output is shown in figure 6. for the testing condition: 10 -fold cross validation balanced dataset for the Naïve Bayes model.

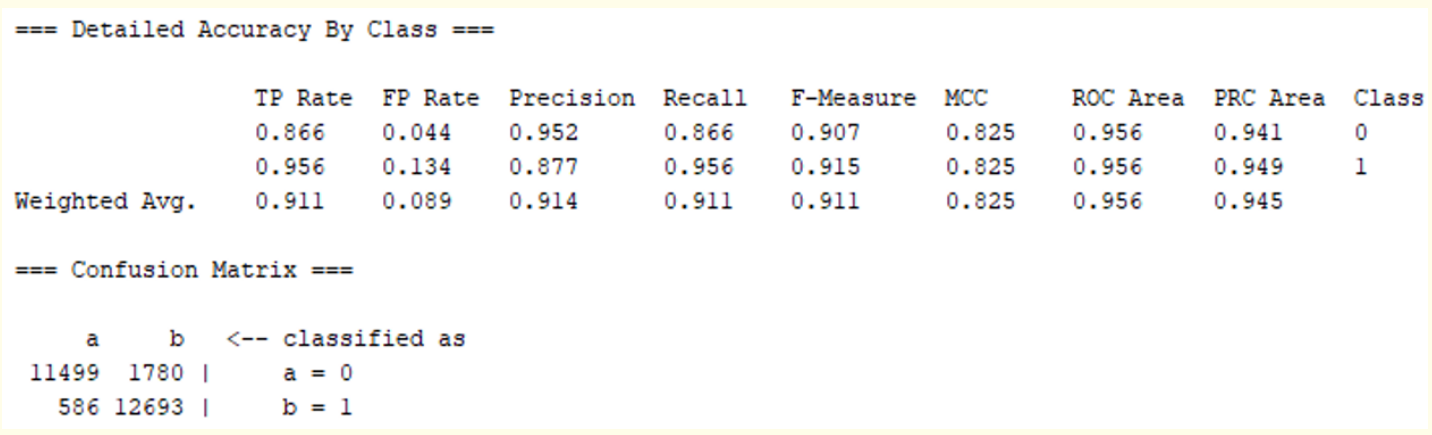

Figure 6: Result of Naïve Bayes Classifier Balanced Dataset 10-Fold Cross-validation.

As shown in figure 6, TP Rate (rate of true positives) is the rate of the cases that correctly classified as the given class and is also known as Sensitivity or Recall. The weighted average Sensitivity considers the size of the category in calculations, and the unweighted average measure does not consider the category size and is a simple average of Sensitivity of two categories. For this testing condition, the weighted Sensitivity was 0.911 and unbalanced weighted Sensitivity was 0.893 (average of Class-0 Sensitivity $=0.866$ and Class-1 Sensitivity=0.956). Similarly, Specificity is presented in the WEKA output as Precision for each category. figure 6 also shows Fmeasure, which is a combined measure of sensitivity and specificity, calculated as their harmonic mean. For this study, we examined Sensitivity, Sensitivity, F-measure to assess the prediction performance.

The Sensitivity and Specificity of Naïve Bayes model for all different testing conditions are presented in figures 7 and 8 respectively. As shown in figure 7, the Sensitivity of Class 0 for unbalanced dataset is higher than for the balanced dataset under different testing conditions. This could be probably because, Class 0 is the highly dominant category in the unbalanced dataset and thus predicted well, but not so in the balanced dataset. The sensitivity and specificity values of Class 1 differ considerably for the balanced and unbalanced datasets under different testing conditions, with the prediction performance being significantly better for the balanced dataset as it is an extremely small category in the unbalanced dataset. It is to be noted that for the unbalanced dataset, the weighted average Sensitivity and Specificity closely follow the Class 0 values as it is the heavily dominant category.

As shown in figures 7 and 8 for Naïve Bayes classifier, over the five different testing conditions, the $90 \%$ percentage training had the best prediction result for unbalanced dataset where it had the best Sensitivity and Specificity values for class 1 prediction and overall great measurements for class 0 prediction. The 10 -fold cross-validation observed the best result for balanced dataset, which had great values for all Sensitivity, Specificity, and F-measure for both classes. Comparing the best results of balanced and unbalanced datasets, balanced dataset had averaged good prediction performance for both class 1 and class 0 , whereas unbalanced dataset only predicted well for class 0 but not class 1 . For example, for Class 1, the highest Specificity and F-measure were only 0.432 and 0.542 for the unbalanced dataset; however, the highest Specificity and F-measure were 0.877 and 0.915 for the balanced dataset. The highest weighted unbalanced F-measure was 0.905 and balanced F-measure was 0.911, which indicates that the overall weighted performance was similar for balanced and unbalanced datasets and it does not highlight the poor prediction performance of Class 1 in the unbalanced dataset. 


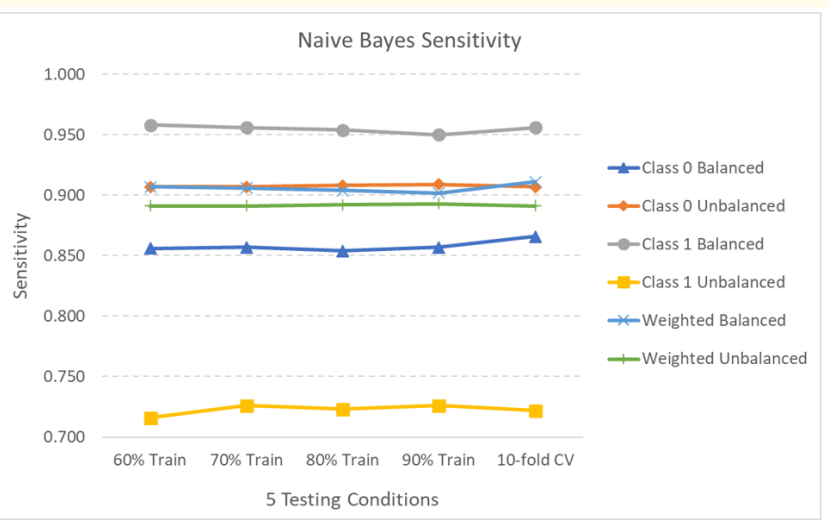

Figure 7: Naïve Bayes Classifier Sensitivity.

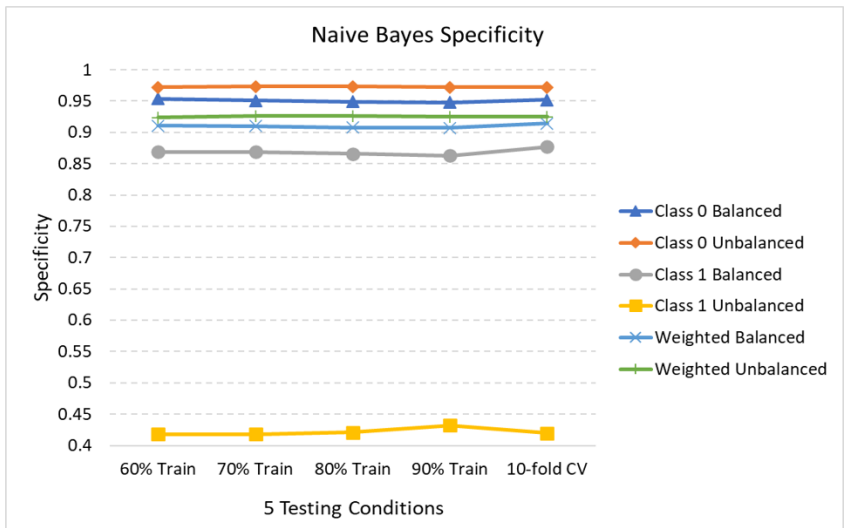

Figure 8: Naïve Bayes Classifier Specificity.

\section{Logistic regression}

The Sensitivity and Specificity of Logistic Regression model for all different testing conditions are presented in figures 9 and 10 respectively. For Logistic Regression, among the five different testing conditions, 10 -fold cross-validation option performed the best result for unbalanced dataset with the overall better measurements of Sensitivity, Specificity, and F-measure for both classes; the $70 \%$ percentage split option predicted the best result for unbalanced dataset, where it had better class 1 prediction and the best weighted results than other four conditions. Comparing the balanced and unbalanced conditions, balanced dataset again had better overall performance than unbalanced dataset with better Sensitivity (0.961) and Specificity (0.899).

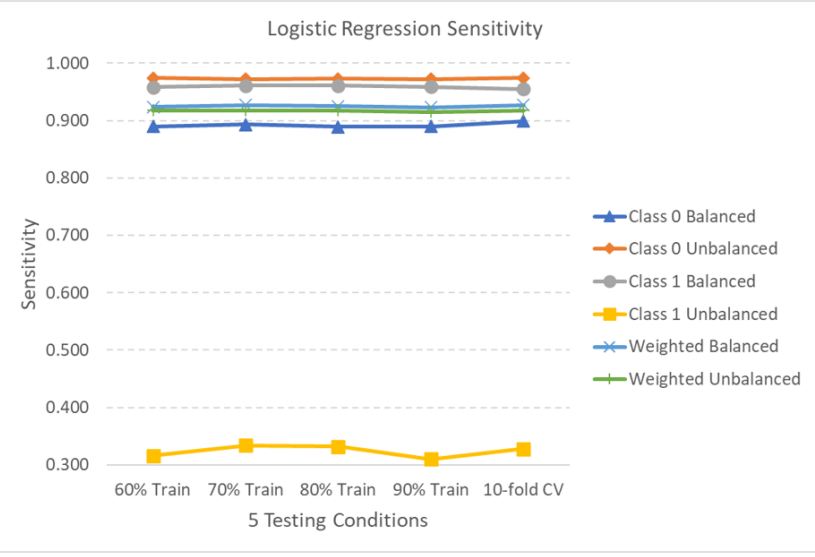

Figure 9: Logistic Regression Sensitivity.

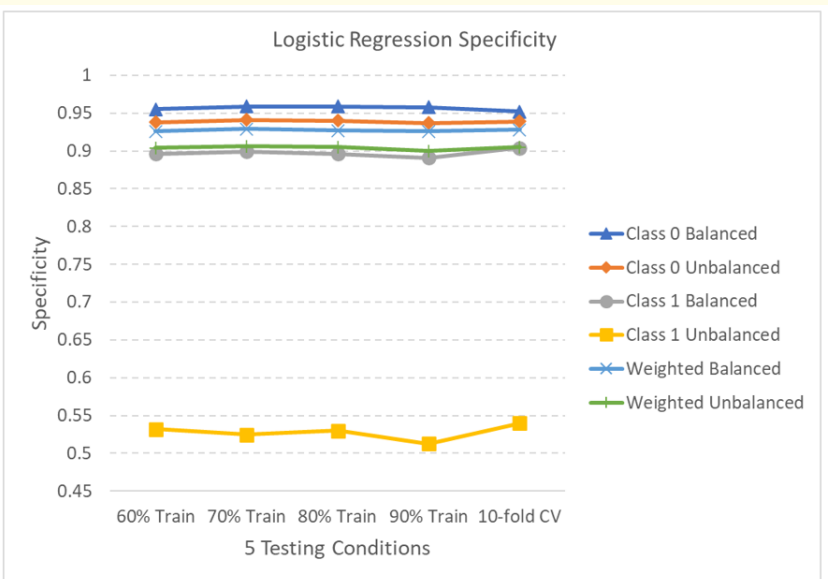

Figure 10: Logistic Regression Specificity.

\section{Support vector machine}

The results of the Support Vector Machine for model for all different testing conditions are shown in figures 11 and 12, respectively. For balanced dataset with Support Vector Machine (SVM), the prediction results did not change much over the five different testing conditions, which means that the measurements of Sensitivity, Specificity, and F-measure were similar. As for the unbalanced dataset, the $70 \%$ percentage split option had the best prediction performance. Compared these two results, balanced dataset still predicted better than unbalanced just like the previous three algorithms, especially due to low sensitivity of the unbalanced class 1 result (recall $=0.293$ ). 


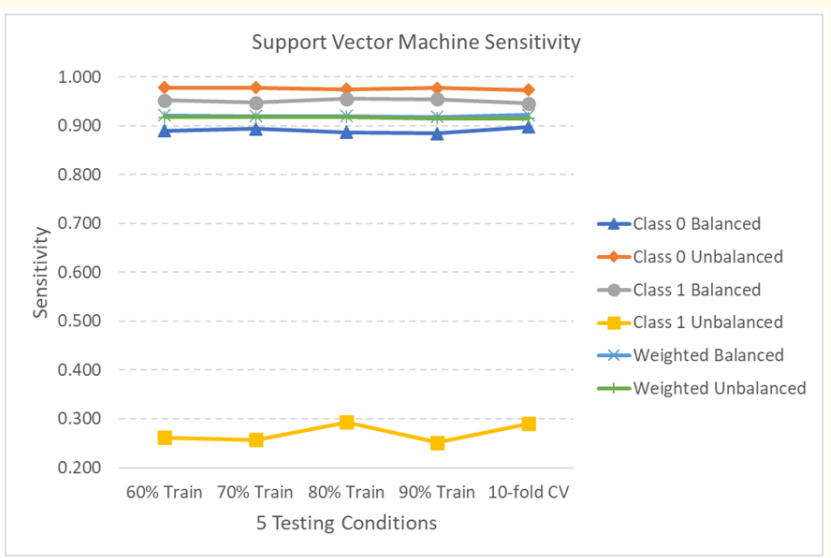

Figure 11: Support Vector Machine Sensitivity.

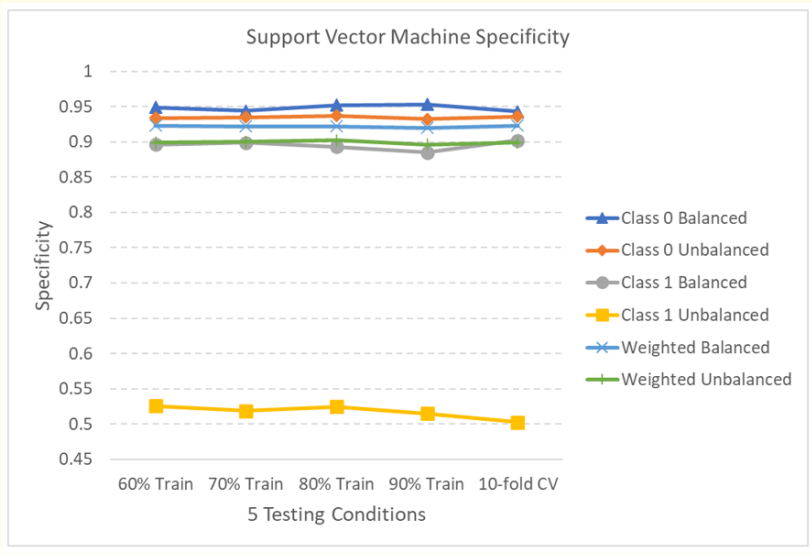

Figure 12: Support Vector Machine Specificity.

\section{Multilayer perceptron}

The results of the Multilayer Perceptron algorithm for both balanced and unbalanced datasets are shown in figures 13 and 14 respectively. For Multilayer Perceptron method, unbalanced dataset performed the best with the $80 \%$ percentage split testing option, while balanced dataset had the best prediction when it was with folds 10 cross-validation testing condition. Comparing the balanced and unbalanced conditions, similar to the Naïve Bayes Classifier, balanced dataset predicted better for both two classes, but unbalanced dataset only predicted well for class 0 . The low percentage of sensitivity (0.401), specificity (0.537), and F-measure $(0.459)$ of unbalanced prediction made the unbalanced dataset had worse weighted results than balanced dataset.

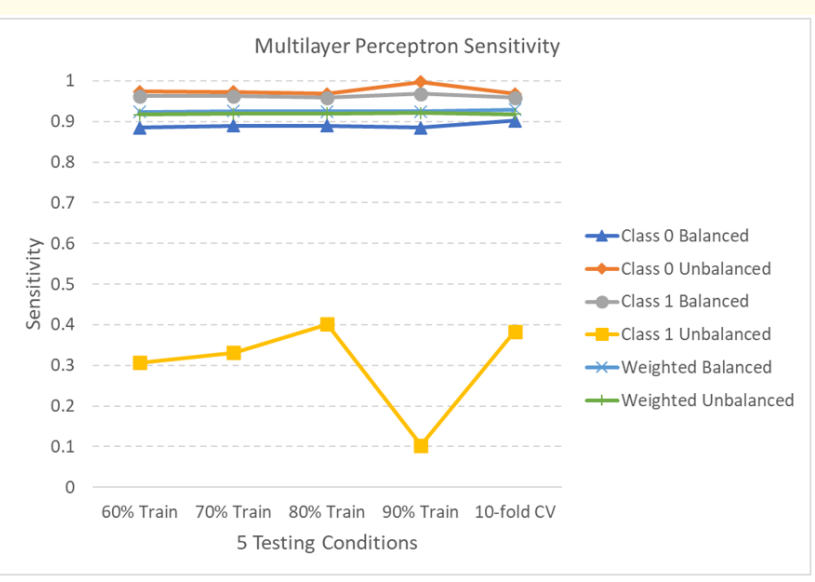

Figure 13: Multilayer Perceptron Sensitivity.

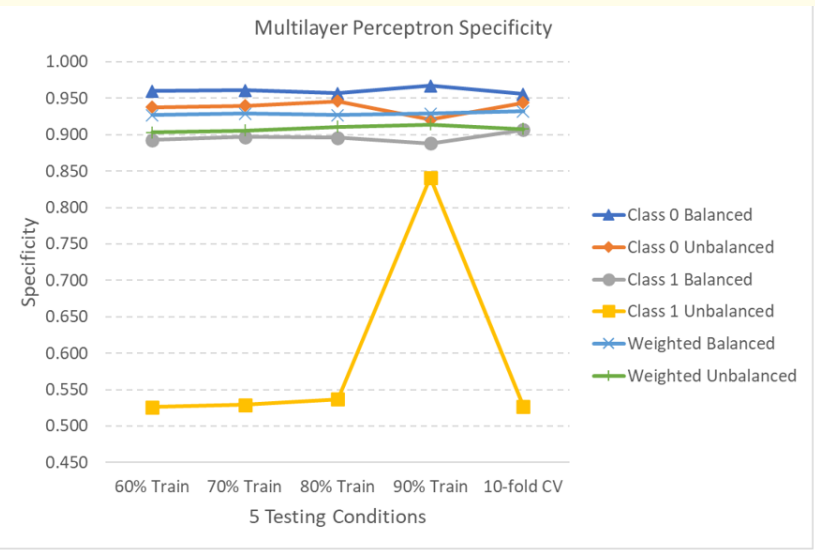

Figure 14: Multilayer Perceptron Specificity.

\section{Comparative analysis}

The prediction results in terms of Sensitivity and Specificity are summarized for each model under different testing conditions in table 2 .

As shown in table 2, for all the four algorithms-Naïve Bayes, MLP, Logistic Regression, and SVM, the predictions of the balanced dataset were much better than unbalanced dataset. All the performance measures (Sensitivity and Specificity) for class 1 for unbalanced dataset were much worse than class 0 , which also made the weighted average results relatively lower as compared to the corresponding balanced dataset testing condition. Primarily this hap- 


\begin{tabular}{|c|c|c|c|c|c|c|c|c|}
\hline \multirow{2}{*}{ Model } & \multirow{2}{*}{ Dataset } & \multirow{2}{*}{$\begin{array}{c}\text { Test Condi- } \\
\text { tion }\end{array}$} & \multicolumn{2}{|c|}{ Class 0} & \multicolumn{2}{|c|}{ Class 1} & \multicolumn{2}{|c|}{ Weighted } \\
\hline & & & Sensitivity & Specificity & Sensitivity & Specificity & Sensitivity & Specificity \\
\hline \multirow[t]{10}{*}{ Naïve Bayes } & \multirow[t]{5}{*}{ Balanced } & 10 -fold & 0.866 & 0.952 & 0.956 & 0.877 & 0.911 & 0.914 \\
\hline & & $60 \%$ & 0.856 & 0.954 & 0.958 & 0.869 & 0.907 & 0.911 \\
\hline & & $70 \%$ & 0.857 & 0.951 & 0.956 & 0.869 & 0.906 & 0.910 \\
\hline & & $80 \%$ & 0.854 & 0.949 & 0.954 & 0.866 & 0.904 & 0.908 \\
\hline & & $90 \%$ & 0.857 & 0.948 & 0.950 & 0.863 & 0.902 & 0.907 \\
\hline & \multirow[t]{5}{*}{ Unbalanced } & 10 -fold & 0.907 & 0.972 & 0.722 & 0.420 & 0.891 & 0.925 \\
\hline & & $60 \%$ & 0.907 & 0.972 & 0.716 & 0.418 & 0.891 & 0.924 \\
\hline & & $70 \%$ & 0.907 & 0.973 & 0.726 & 0.418 & 0.891 & 0.926 \\
\hline & & $80 \%$ & 0.908 & 0.973 & 0.723 & 0.421 & 0.892 & 0.926 \\
\hline & & $90 \%$ & 0.909 & 0.972 & 0.726 & 0.432 & 0.893 & 0.925 \\
\hline \multirow{10}{*}{$\begin{array}{l}\text { Logistic } \\
\text { Regression }\end{array}$} & \multirow[t]{5}{*}{ Balanced } & 10 -fold & 0.899 & 0.952 & 0.955 & 0.904 & 0.927 & 0.928 \\
\hline & & $60 \%$ & 0.890 & 0.955 & 0.958 & 0.896 & 0.924 & 0.926 \\
\hline & & $70 \%$ & 0.893 & 0.959 & 0.961 & 0.899 & 0.927 & 0.929 \\
\hline & & $80 \%$ & 0.889 & 0.959 & 0.961 & 0.896 & 0.925 & 0.927 \\
\hline & & $90 \%$ & 0.890 & 0.958 & 0.959 & 0.891 & 0.923 & 0.926 \\
\hline & \multirow[t]{5}{*}{ Unbalanced } & 10-fold & 0.974 & 0.939 & 0.328 & 0.540 & 0.918 & 0.905 \\
\hline & & $60 \%$ & 0.974 & 0.938 & 0.316 & 0.532 & 0.918 & 0.904 \\
\hline & & $70 \%$ & 0.972 & 0.941 & 0.334 & 0.525 & 0.918 & 0.906 \\
\hline & & $80 \%$ & 0.973 & 0.940 & 0.332 & 0.530 & 0.918 & 0.905 \\
\hline & & $90 \%$ & 0.972 & 0.937 & 0.310 & 0.513 & 0.915 & 0.900 \\
\hline \multirow[t]{10}{*}{ SVM } & \multirow[t]{5}{*}{ Balanced } & 10 -fold & 0.898 & 0.943 & 0.946 & 0.902 & 0.922 & 0.923 \\
\hline & & $60 \%$ & 0.890 & 0.949 & 0.952 & 0.896 & 0.921 & 0.923 \\
\hline & & $70 \%$ & 0.894 & 0.944 & 0.947 & 0.899 & 0.920 & 0.922 \\
\hline & & $80 \%$ & 0.887 & 0.952 & 0.955 & 0.893 & 0.920 & 0.922 \\
\hline & & $90 \%$ & 0.884 & 0.953 & 0.954 & 0.885 & 0.918 & 0.920 \\
\hline & \multirow[t]{5}{*}{ Unbalanced } & 10 -fold & 0.973 & 0.936 & 0.290 & 0.503 & 0.915 & 0.899 \\
\hline & & $60 \%$ & 0.978 & 0.934 & 0.262 & 0.526 & 0.917 & 0.899 \\
\hline & & $70 \%$ & 0.978 & 0.935 & 0.257 & 0.519 & 0.917 & 0.900 \\
\hline & & $80 \%$ & 0.975 & 0.937 & 0.293 & 0.525 & 0.918 & 0.902 \\
\hline & & $90 \%$ & 0.977 & 0.932 & 0.252 & 0.515 & 0.915 & 0.896 \\
\hline \multirow{10}{*}{$\begin{array}{l}\text { Multilayer } \\
\text { Perceptron }\end{array}$} & \multirow[t]{5}{*}{ Balanced } & 10 -fold & 0.902 & 0.956 & 0.959 & 0.907 & 0.930 & 0.932 \\
\hline & & $60 \%$ & 0.885 & 0.960 & 0.963 & 0.893 & 0.924 & 0.927 \\
\hline & & $70 \%$ & 0.890 & 0.961 & 0.963 & 0.897 & 0.926 & 0.929 \\
\hline & & $80 \%$ & 0.890 & 0.957 & 0.959 & 0.896 & 0.925 & 0.927 \\
\hline & & $90 \%$ & 0.885 & 0.967 & 0.968 & 0.888 & 0.925 & 0.929 \\
\hline & \multirow[t]{5}{*}{ Unbalanced } & 10 -fold & 0.968 & 0.944 & 0.383 & 0.527 & 0.918 & 0.908 \\
\hline & & $60 \%$ & 0.974 & 0.938 & 0.307 & 0.526 & 0.917 & 0.903 \\
\hline & & $70 \%$ & 0.973 & 0.940 & 0.331 & 0.529 & 0.919 & 0.906 \\
\hline & & $80 \%$ & 0.968 & 0.946 & 0.401 & 0.537 & 0.920 & 0.911 \\
\hline & & $90 \%$ & 0.998 & 0.921 & 0.103 & 0.841 & 0.921 & 0.914 \\
\hline
\end{tabular}

Table 2: Summary of prediction performance of different machine learning models. 
pened due to the heavy bias in the data between class 0 and class 1 in the unbalanced dataset. When the considered number of records of class 0 and class 1 were the same in the balanced data with no bias present, the prediction results were comparatively better.

Overall, for both unbalanced and balanced dataset, Multilayer Perceptron (MLP) method performed the best over the four classifiers, whereas Naïve Bayes classifier performed the worst compared to the other three. For the unbalanced dataset using MLP, the overall true positive rate (TPR) was 0.920 (weighted recall); true negative rate (TNR) was 0.911 (weighted precision); and the F-measure of the model was 0.915 . For the balanced dataset over MLP, the overall true positive rate (TPR) was 0.930 (weighted recall); true negative rate (TNR) was 0.932 (weighted precision); and the F-measure of the model was 0.930. As for the Naïve Bayes classifier, the best unbalanced sensitivity was 0.893 (weighted recall); the best specificity was 0.925 (weighted precision); and the best weighted f-measure was 0.905 . For the balanced dataset over Naïve Bayes, the overall sensitivity was 0.911 (weighted recall); the overall specificity was 0.914 (weighted precision); and the weighted fmeasure of the model was 0.911 .

\section{Conclusion}

In this study, the impact of bias in data on accurate prediction of likelihood of breast cancer was examined, based on biographical and diagnostic data by evaluating the prediction performance of four widely used machine learning models on the BCSC cancer dataset with 154,899 cases including 11 different features for the prediction of the likelihood of breast cancer. The original dataset was used to create balanced and unbalanced datasets to study the impact of bias by comparing the performances of the four machine learning algorithms of Naïve Bayes classifier, Logistic Regression, Multilayer Perceptron, and Support Vector Machine on the balanced and unbalanced dataset under five different testing conditions.

Overall, Multilayer Perceptron method predicted the best among these four algorithms, whereas Naïve Bayes classifier performed the worst for both unbalanced and balanced datasets. Also, the prediction from the balanced dataset for all four algorithms were always better than the prediction from the unbalanced dataset. This phenomenon meant that having the same number of cases for both class 1 and class 0 in the dataset when training the model can avoid the bias and improve the prediction performance of the model.
Comparing the prediction performances over balanced and unbalanced training datasets, on the one hand, unbalanced training dataset did a better job ruling out the women, who didn't have breast cancer, but being too cautious in finding cases that had breast cancer compared to the balanced dataset. On the other hand, the prediction of the balanced training dataset missed fewer breast cancer cases, but falsely over-predicted of the patients who did not have breast cancer than unbalanced dataset.

The consistent gap in prediction performance of various machine learning models suggest that the bias in the data negatively impacts the prediction performance of positive cancer cases leading to poor Sensitivity and Specificity for Class 1 . Future studies should examine different approaches to address this issue of unbalance in data for predicting breast cancer likelihood.

\section{Acknowledgements}

We are thankful for the BCSC dataset provided by the National Cancer Institute.

\section{Conflict of Interest}

None.

Bibliography

1. "Breast Cancer Statistics". Centers for Disease Control and Prevention (2021).

2. Siegel R L., et al. "Cancer Statistics, 2015”. CA: A Cancer Journal for Clinicians 65.1 (2015): 5-29.

3. Siegel R L., et al. "Cancer Statistics, 2016". CA: A Cancer Journal for Clinicians 66.1 (2016): 7-30.

4. U.S. Breast Cancer Statistics (2021).

5. Siegel R L., et al. "Cancer Statistics, 2019”. CA: A Cancer Journal for Clinicians 69.1 (2019): 7-34.

6. Sun Y., et al. "Risk Factors and Preventions of Breast Cancer". International Journal of Biological Science 13.11 (2017): 13871397.

7. Al-Khasawneh A. "Diagnosis of Breast Cancer Using Intelligent Information Systems Techniques". International Journal of EHealth and Medical Communications 7.1 (2016): 65-75.

8. Listgarten J., et al. "Predictive Models for Breast Cancer Susceptibility from Multiple Single Nucleotide Polymorphisms". Clinical Cancer Research 10.8 (2004): 2725-2737. 
9. Chen Y C., et al. "Risk classification of cancer survival using ANN with gene expression data from multiple laboratories". Computers in Biology and Medicine 48 (2014): 1-7.

10. Akay M F. "Support vector machines combined with feature selection for breast cancer diagnosis". Expert Systems with Applications 36.2 (2009): 3240-3247.

11. Park K., et al. "Robust predictive model for evaluating breast cancer survivability". Engineering Applications of Artificial Intelligence 26.9 (2013): 2194-2205.

12. Hastie T. "The Elements of Statistical Learning: Data Mining, Inference and Prediction ( $2^{\text {nd }}$ Edition)". Springer New York (2009).

13. Kononenko I. "Machine learning for medical diagnosis: History, state of the art and perspective". Artificial Intelligence in Medicine 23.1 (2001): 89-109.

14. Cruz J A., et al. "Applications of Machine Learning in Cancer Prediction and Prognosis". Cancer Informtics 2 (2006): 59-77.

15. Pironet A., et al. "Machine Learning-Based Extraction of Breast Cancer Receptor Status From Bilingual Free-Text Pathology Reports". Frontiers in Digital Health 3 (2021): 102.

16. Ming C., et al. "Machine learning techniques for personalized breast cancer risk prediction: comparison with the BCRAT and BOADICEA models". Breast Cancer Research 21.1 (2019).

17. Asri H., et al. "Using Machine Learning Algorithms for Breast Cancer Risk Prediction and Diagnosis". Procedia Computer Science 83 (2016): 1064-1069.

18. Li M., et al. "Machine Learning-Based Decision Support System for Early Detection of Breast Cancer". Indian Journal of Pharmaceutical Education and Research 54.3 (2020): 705-715.

19. Ahmad L G., et al. "Using Three Machine Learning Techniques for Predicting Breast Bancer Recurrence". Journal of Health and Medical Informatics 4.2 (2013): 2.

20. Breast Cancer Surveillance Consortium. (HHSN261201100031C) (2021).

21. Rajendran K., et al. "Predicting Breast Cancer via Supervised Machine Learning Methods on Class Imbalanced Data". International Journal of Advanced Computer Science and Applications 11.8 (2020): 54-63.

22. Mitchell T. "Machine Learning”. McGraw-Hill (1997).
23. Park Y S and Lek S. "Chapter 7 - Artificial Neural Networks: Multilayer Perceptron for Ecological Modeling”. In S. E. Jorgensen (Eds), Developments in Environmental Modelling 28 (2016): 123-140.

24. Gupta P and Sinha N K. "CHAPTER 14 - Neural Networks for Identification of Nonlinear Systems: An Overview". In N. K. Sinha and M. M. Gupta (Eds), Soft Computing and Intelligent Systems (2000): 337-356.

25. Ahmad L G., et al. "Using Three Machine Learning Techniques for Predicting Breast Bancer Recurrence". Journal of Health and Medical Informatics 4.2 (2013): 2.

26. Delen D., et al. "Predicting breast cancer survivability: a comparison of three data mining methods". Artificial Intelligence in Medicine 34.2 (2005): 113-127.

\section{Assets from publication with us}

- Prompt Acknowledgement after receiving the article

- Thorough Double blinded peer review

- Rapid Publication

- Issue of Publication Certificate

- High visibility of your Published work

Website: www.actascientific.com/

Submit Article: www.actascientific.com/submission.php

Email us: editor@actascientific.com

Contact us: +919182824667 